\title{
=ALTERIDAD Perspectiva de género en Educación Básica Superior y Bachillerato
}

\section{Gender perspective in Superior Basic Education and High School}

D Ximena Patricia Vizuete-Salazar es docente e investigadora de la Pontificia Universidad Católica del Ecuador y de la Unidad Educativa Amelia Gallegos Díaz (Ecuador) (ximena.p.vizuete.s@pucesa.edu.ec) (https://orcid.org/0000-0003-2591-4215)

(D) Dr. Alexander Ramón Lárez-Lárez es docente e investigador de la Pontificia Universidad Católica del Ecuador, Universidad de Otavalo (Ecuador) (alarez@pucesa.edu.ec) (https://orcid.org/0000-0003-2763-5113)

Recibido: : 2020-08-21 / Revisado: 2020-11-20 / Aceptado: 2020-12-08 / Publicado: 2021-01-01

\section{Resumen}

Investigaciones sobre género han demostrado que el ambiente escolar es el espacio idóneo para eliminar estereotipos sobre desigualdad, discriminación y violencia. Esto se debe a que, es en este periodo, donde los adolescentes aprenden a relacionarse según sus concepciones culturales o siguiendo estereotipos aprendidos desde su hogar o en el entorno. Consecuentemente, el estudio tuvo como objetivo evaluar la influencia de la perspectiva de género en la interacción social del adolescente en ambientes escolares en los niveles de Educación General Básica Superior y Bachillerato en la Unidad Educativa Amelia Gallego Díaz. La investigación se fundamentó en un enfoque cuantitativo, diseño no experimental, corte transversal y alcance descriptivo. El instrumento utilizado para la recogida de datos ha sido una adaptación de la escala de actitudes hacia la igualdad de género dirigido al alumnado de García-Pérez et al. (20 I0), aplicado a una muestra de 263 estudiantes de la institución en estudio. Al hacer el análisis, los resultados en los indicadores de la perspectiva de género muestran una actitud adaptativa que incide directamente en un trato de igualdad y equidad en la interacción social de los estudiantes; es decir, en esta institución educativa los estudiantes se han ido adaptando a las situaciones que la actualidad les depara, sin embargo, aún queda un importante porcentaje que se mantiene firme a las enseñanzas que les han inculcado en su núcleo familiar.

Descriptores: Ambiente educacional, interacción social, perspectiva de género, educación, estereotipos de género, adaptación del estudiante.

\section{Abstract}

Research on gender has shown that the school environment is the ideal space to eliminate stereotypes about inequality, discrimination, and violence. This is because, during this period, adolescent learn to form relations with others based on their cultural conceptions or following stereotypes learned from home or environment. Consequently, the objective of this study was to evaluate the influence gender perspective on the social interaction of adolescents in school environments, in secondary and high school education at Amelia Gallego Díaz high school. This research was carried out with a quantitative approach, non-experimental design, cross-sectional, and descriptive scope. The method employed for data collection was scale adaptation of attitudes towards gender equality aimed at students from García-Pérez et al. (20 I0) was implemented. This was applied to a sample of 263 students from the institution under study. When analysing the data, the results on the indicators, from gender perspective portrayed an adaptive attitude that directly affects equality and impartial treatment in the social interaction of students; this means in this high school, students have been adapting to the situations that nowadays arise. However, there is still a significant percentage of students that remain firm to the disciplines they have instilled from their family nucleus.

Keywords: Educational environment, social interaction, gender perspective, education, gender stereotypes, student adjustment.

Forma sugerida de citar: Vizuete-Salazar, X., \& Lárez-Lárez, A. (2021). Perspectiva de género en Educación Básica Superior y Bachillerato. Alteridad, 16(1), 130-141. https://doi.org/10.17163/alt.v16n1.2021.10 


\section{Introducción}

En la reciente década se han abordado diferentes investigaciones sobre la perspectiva de género, desde diferentes ámbitos (Benavente \& Valdés, 2014, Trejo-Sirvent et al., 2015; Camarera \& Saavedra, 2018). En los mismos se destaca, la atención para mejorar la igualdad y la equidad; disminuir la desigualdad, discriminación y violencia entre hombres y mujeres. Así mismo, países de todo el mundo han incorporado en sus constituciones nacionales, regulaciones sobre la igualdad de género y destinado, parte de sus recursos, a la implementación de políticas que luchan con la desigualdad, la exclusión y discriminación que impulsan la participación femenina en diversos contextos de la sociedad (Organización de las Naciones Unidas, 2014). Aun cuando, se han conseguido grandes avances, continúan apareciendo desigualdades en las sociedades respecto a conductas sexistas, roles de género y reproducción de estereotipos. Al respecto, Duarte y García (2016) refieren que la diferencia entre sexos y la opresión de género se debe a que existen discrepancias biológicas e históricas que no se pueden cambiar, y que se afirman en ideologías o mitos.

Este planteamiento, tal como corrobora Chávez-Carapia (2017), aún no se ha logrado. Múltiples esfuerzos se han desarrollado a nivel internacional, a lo largo de los años. Destaca la IV Conferencia de Beijín (1995) como obligación y deber gubernamental de los suscribientes, establecer una plataforma para encaminar sus políticas en la que se respeten la igualdad de hombres y mujeres. Sin embargo, estudios realizados por organizaciones a favor de los derechos en el mundo, han alertado sobre estudiantes gays, bisexuales, transgéneros y lesbianas que han sido víctimas de agresiones en el entorno de su escuela, mayoritariamente por otros alumnos, directivos y docentes violentos (Organización de las Naciones Unidas para la Educación, la Ciencia y la Cultura, 2015). Lo que evidencia, un largo camino por recorrer.
La problemática persiste a nivel mundial, en Ecuador no es diferente. El género en la sociedad ecuatoriana ha tomado relevancia como una forma para lograr derechos de igualdad en sectores discriminados. La discriminación estigmatiza al ser humano pues lo diferencia, tanto en lo físico como en lo cultural, evidenciando la desigualdad y fijación de estereotipos. La Constitución de la República (2008) incorporó principios y derechos para enfrentar la inequidad, violencia de género y la discriminación. Elementos que también han sido mencionados en el Plan Nacional Toda una Vida (2017-2021) y en el documento emitido por la Defensoría del Pueblo, denominado "Política Institucional de Igualdad de Género 2016-2019”. Al respecto este último organismo menciona que, aun cuando se han dado avances en la materia, el Ecuador tiene una "cultura arraigadamente patriarcal y androcéntrica, reflejada en prácticas sociales e institucionales, que evidencian la ausencia de estrategias y acciones que promuevan la igualdad de género y la no discriminación" (p. 6).

En materia educativa, también es imperioso tratar de erradicar la discriminación a través de la tolerancia y la educación (Medina et al., 2015). Incorporar en la formación el enfoque hacia la perspectiva de género, según Solís (2016), permite transformar esquemas valorativos en los que se desarrollen destrezas y habilidades desde las diferencias sexuales y de género, como parte del proceso de aprendizaje. Para Castillo y Gamboa (2013), "la igualdad de género requiere de un proceso de aculturación en el cambio de las concepciones y prácticas que han sido socialmente aprendidas" (citado en Azorín, 2017, p. 46). Refieren que, el adolescente es un grupo clave de la sociedad, que debe ir formándose, con una perspectiva de género que ejerza una igualdad entre sexos desde los diversos contextos sociales.

Según el Fondo de las Naciones Unidas para la Infancia (UNICEF), la adolescencia es el espacio donde el joven aprende a relacionarse con sus pares, entre hombres y mujeres, según 
sus concepciones culturales o siguiendo estereotipos aprendidos desde su hogar o el entorno (UNICEF, 2015). Es una etapa para iniciar relaciones con otros y definir su identidad: experiencia y conocimiento. Lozano (2014), afirma que el adolescente en el proceso de precisar su personalidad, expandir sus relaciones entre iguales o constituirse parte de un grupo, sigue estereotipos de género de acuerdo con concepciones culturales sobre la que se constituye su formación, partiendo de elementos de su identidad asignados por la cultura. El adolescente aparte de luchar con sus aspectos físicos y emocionales de la edad, también tiene que enfrentarse a situaciones externas como la imposición de la cultura en cuanto al género, que se refleja en la forma de vestir, de expresarse y de relacionarse con sus pares; y seguir patrones de conducta impuestos por la sociedad.

Sustentado en estos planteamientos, la investigación reviste gran importancia en el campo social y especialmente educativo, porque permitió comprender y visibilizar las concepciones de género y estereotipos que están actuando en el lenguaje, comportamiento y otras manifestaciones. Por ello, se planteó como propósito, evaluar cómo la perspectiva de género influye en la interacción social del adolescente en ambientes escolarizados, en los niveles de Educación General Básica Superior y Bachillerato de la Unidad Educativa Amelia Gallegos Díaz, ubicada en Riobamba-Ecuador. A partir de la identificación de los comportamientos basados en estereotipos de género del adolescente, se procedió a determinar y analizar los factores que inciden, en esta perspectiva, para el desarrollo social dentro de espacios escolares y establecer los niveles de incidencia entre las dos dimensiones (perspectiva de género e interacción social).

\subsection{Concepciones de género}

El enfoque de género en la adolescencia, como temática de investigación, ha tomado relevancia. Rodríguez et al. (2017) afirman que el género representa un elemento propio e intrínseco de la personalidad, donde se conjugan creencias, estilo de vida, oportunidades y el comportamiento en la sociedad. Guzmán (2015) señala que existe un papel de supremacía y poder de los hombres sobre las mujeres que se basa en la forma dicotómica y estructural en que las personas construyen el mundo. Los términos sexo y género son diferentes. Según Liscano (2016) el sexo es la visión biológica que diferencia a hombres y mujeres, mientras que el género es la dimensión sociocultural de las conductas, maneras y expresiones de las personas. Guerra López (2016) menciona que el género va creando un perfil social de los individuos de acuerdo con su naturaleza o el sexo al que pertenecen. Estos planteamientos permiten afirmar que el sexo y el género están relacionados, pero cada uno cumple una función específica; mientras que el primero está determinado por una condición desde que el sujeto nace, el segundo se caracteriza por la influencia de la sociedad. Así, de manera universal, se encuentran concepciones de las actuaciones y papeles que hombres y mujeres tienen dentro de la sociedad. De acuerdo con Torres (2018), estas manifestaciones están condicionadas e identificadas por su naturaleza de ser y hacer en la esfera social; desde el nacimiento, la representación social y sexual masculina y femenina estará predeterminado, independientemente del hecho de que, como persona madura, no quiera cumplir con la tarea que se le ha encomendado.

De igual forma, Serret (2016) expresa que hombres y mujeres no son iguales, pues biológicamente, es así, sino también porque son definidos cada uno, como consecuencia de lo que aprenden en su entorno ideológico, familiar, económico y social. Entonces, los roles de género se basan en el sexo y son las normas sociales y de comportamiento, percibidas socialmente como apropiadas y generalizadas históricamente. Esta representación social de hombres y mujeres para Bruel et al. (2013), se fundamenta en el comportamiento propio de cada uno y la interacción entre individuos. Para Aguilar et al. (2013), la 
diferencia radica en la formación de ambos para ejercer su función en la sociedad: el hombre se considera independiente, autónomo y con poder, mientras que la mujer es educada para atender y cuidar a las personas; guardiana del hogar.

El comportamiento de los jóvenes en la adolescencia tiene particularidades al asumir un rol en la sociedad. Beltrán (2012) afirma que los hombres adolescentes, en muchos casos como una forma de demostrar su masculinidad, adquieren prácticas culturales tales como que el hombre tiene que defenderse, hacer deporte, usar determinado estilo de peinado, vestimenta, uso de lenguaje grotesco e insultante, etc. En esta etapa de desarrollo social del adolescente se adquieren actitudes y valores que ayudan a la formación de su identidad para el manejo de conflictos y la regulación de la agresión. Según Javaloy et al. (2016), es una forma de mantener la sociedad a través del intercambio de expresiones, palabras, gestos en los que las personas participan con un objetivo común. Para Uranga et al. (2016), se considera la relación con otros individuos, que es parte de la vida de los seres, en la cual surgen las creencias humanas y, a través de este vínculo, se moldean las actitudes y se crean valores. Desde una perspectiva similar, Suárez y Vélez (2016) exponen que es una forma de dar orden a los comportamientos para relacionarse con otras personas e influir en aquellos con quienes se interactúa desde una determinada perspectiva. Siguiendo a Meza y Páez (2016) es una influencia recíproca en la que tanto el individuo como las demás personas, interactúan, cambian o modifican el comportamiento.

Por lo tanto, el contexto educativo le dará la oportunidad de discernir, si la educación que ha recibido le ayudará a adaptarse al entorno social, o si, por el contrario, tendrá que hacer algunos cambios en el comportamiento y la conducta. Durante este período, que va desde la niñez hasta la adolescencia, las personas experimentan diferentes tendencias de relaciones que conducen a estilos como el agresivo, asertivo y el pasivo. Estas variaciones dependen del núcleo familiar y su composición. Para Ortiz et al. (2018) la convivencia permite el desarrollo de la empatía en la que intervienen el apego, la reciprocidad afectiva y la construcción de criterios morales sólidos.

Teniendo en cuenta que, la perspectiva de género se vuelca hacia las personas que muestran diversas circunstancias, se puede llegar a comprender que existe una biodiversidad social, donde existe una diferencia sexual y representaciones sociales construidas alrededor de ella. En este sentido, Solís (2016) expone que la:

Perspectiva de género es una visión crítica, explicativa y alternativa de lo que acontece en el orden de género, permitiendo analizar las profundas y complejas causas de dicha opresión y de los procesos históricos que la originan y la reproducen. (pp.101-102)

\section{Metodología}

\subsection{Diseño}

El enfoque de esta investigación se fundamenta en un paradigma cuantitativo; diseño descriptivo, de corte transversal. El método cuantitativo sirvió para recolectar la información, a partir de la cuantificación numérica y análisis estadístico (Cabezas et al., 2018). La descripción permitió evidenciar la realidad directamente e identificar los factores que incidieron en la perspectiva de género, en un ambiente educativo. La investigación fue de corte transversal pues se conoció la realidad en un espacio y tiempo determinado (Hernández et al., 2014).

\subsection{Población y muestra}

La población estuvo conformada por 764 estudiantes de la Unidad Educativa Amelia Gallegos Díaz, de los cuales $510(66,75 \%)$ pertenecieron al sexo femenino y $254(33,25 \%)$, al sexo masculino; distribuidos en paralelos, grados, edades y sexo. La muestra estuvo estructurada por 263 
alumnos de $8^{\circ}, 9^{\circ}$ y $10^{\circ}$ de Educación Básica Superior (entre 12 y 14 años de edad) y $1^{\circ}, 2^{\circ}$ y $3^{\circ}$ de Bachillerato. De acuerdo con el nivel educativo un $40,7 \%$ fueron estudiantes de Básica Superior y un 59,3\% de Bachillerato. Se aplicó un muestreo aleatorio estratificado proporcional, por cuanto la población no fue homogénea (Gamboa, 2017) y se seleccionaron elementos de cada subgrupo. Luego se aplicó un muestreo aleatorio simple por sexo, grados y paralelos y se utilizó la tabla de números aleatorios para seleccionar a cada uno de los participantes.

\subsection{Instrumento}

Un cuestionario fue empleado para la recogida de la información. El mismo se adaptó de la escala de actitudes hacia la igualdad de género, elaborado por García et al. (2010), compuesto por 41 preguntas cerradas, con cuatro opciones de respuesta (nunca, algunas veces, casi siempre y siempre). La confiabilidad fue calculada a través del coeficiente Alfa de Cronbach, y con el software estadístico SPSS versión 21. El resultado fue de 0,801 (confiable).

El instrumento fue organizado en función a los indicadores de las variables: perspectiva de género (estereotipos, discriminación, violencia y sexo) e interacción social (equidad e igualdad). Los ítems 1-9: estereotipos, aborda comportamientos generalizados de hombres y mujeres; ítems 10-16: discriminación, describe diversas situaciones en la que las personas desde su género se sienten excluidas; ítems 17-22: violencia, preguntas relacionadas con agresión, desorden, infidelidad; ítems 23-31: sexo, plantea situaciones en las concepciones de ser hombre y ser mujer; ítems 31-36: equidad, expone interrogan- tes sobre las oportunidades que tienen hombres y mujeres; ítems 37-42): igualdad, indica diversas situaciones en ambientes escolares.

\subsection{Procedimiento}

Inicialmente, se realizaron los trámites respectivos ante la autoridad del Distrito Educativo y de la institución para la debida autorización y se comunicaron los objetivos de la investigación. Previo a la aplicación del instrumento se gestionó la aceptación de los padres de familia y la disposición de los participantes. Se aplicó el instrumento, en el tercer parcial del año lectivo 2019-2020, en horario académico. Finalmente, se procedió a analizar estadísticamente los datos aportados por los involucrados.

\subsection{Análisis de datos}

Para analizar la información se utilizó la estadística descriptiva como promedios, porcentajes y la elaboración de tablas y la estadística inferencial como el análisis de correlación de Spearman's, en función a los valores obtenidos de las respuestas para cada uno de los indicadores, dimensiones y variables.

\section{Resultados}

En la tabla 1 se presentan los puntos de corte utilizados para determinar la actitud de los estudiantes hacia la igualdad de género (sexista, adaptativa o igualitaria) y, a su vez, evaluar la perspectiva de género en la interacción social de los adolescentes en ambientes escolares, niveles educativos de General Básica Superior y Bachillerato, en función de las variables de la investigación. 
Tabla 1. Resultados globales

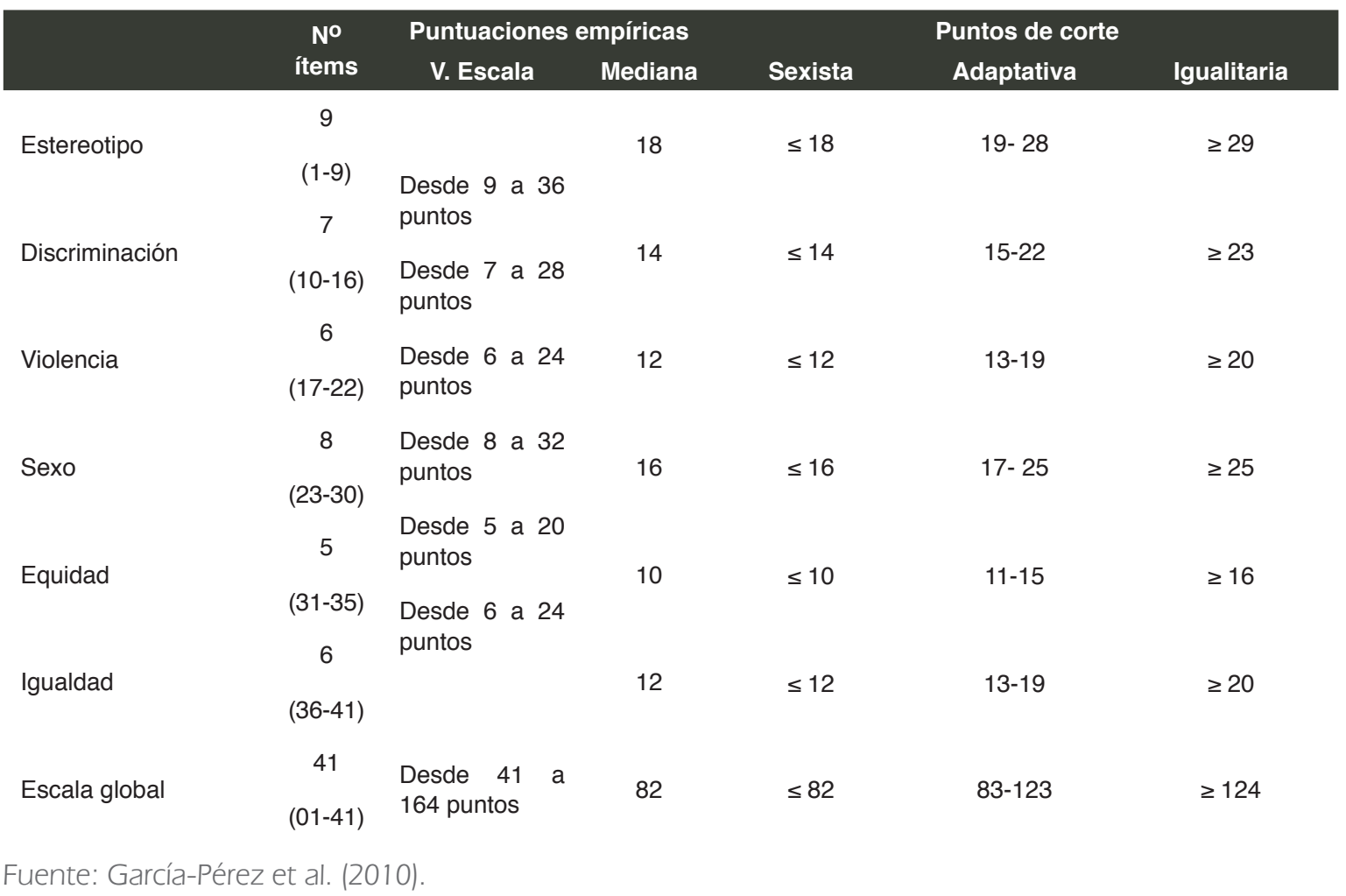

Según García-Pérez et al. (2010) la actitud sexista comprende analizar los puntos de vista competitivo entre hombres y mujeres, así como acciones inconscientes de conductas asociadas al género y modelos sociales donde predomina el hombre sobre la mujer. La actitud adaptativa comprende ejercer acciones fundamentadas en una perspectiva de comportamiento adecuado socialmente que, aun cuando evidencia diferencias y desigualdades, muestra incertidumbre en la valoración de algunos estereotipos. La actitud igualitaria asume la existencia de desigualdad y pondera positivamente las conductas de hombres y mujeres desde una visión con equidad.

En la tabla 3 se puede apreciar el comportamiento de los diferentes indicadores en relación con los puntos de cortes establecidos en la tabla 2. En correspondencia con el estereotipo se puede apreciar una actitud adaptiva en las hembras como los varones. Asimismo, ocurre con la discriminación, la violencia, el sexo, la equidad e igualdad, donde los valores promedios para ambos sexos, expresan una actitud adaptativa hacia la igualdad de género. La perspectiva de los participantes en el estudio basada en los puntos de corte de escala, demuestra que las hembras adoptan actitudes adaptativas $(86,2 \%)$, igualitario $(13,3 \%)$ y sexistas $(0,5 \%)$; mientras que los varones manifiestan actitudes adaptativas $(85,9 \%)$ e igualitario $(14,1 \%)$. Las opiniones de los estudiantes se acercan a niveles próximos hacia la actitud adaptativa (figura 1). 
Tabla 2. Resultados globales en función al sexo

\begin{tabular}{|c|c|c|c|c|}
\hline & \multicolumn{4}{|c|}{ Resultados de la actitud obtenida por los estudiantes en función al sexo } \\
\hline & \multicolumn{2}{|c|}{ Media } & \multicolumn{2}{|c|}{ D.T. } \\
\hline & H & v & H & v \\
\hline Global (42 ítems) & 110,57 & 110,17 & 10,658 & 11,022 \\
\hline Estereotipos & 27,96 & 26,47 & 4,108 & 4,525 \\
\hline Discriminación & 18,66 & 19,39 & 3,664 & 2,915 \\
\hline Violencia & 15,88 & 17,16 & 2,605 & 3,004 \\
\hline Sexo & 19,55 & 19,83 & 3,100 & 3,190 \\
\hline Equidad & 12,62 & 12,05 & 2,247 & 2,705 \\
\hline Igualdad & 15,91 & 15,28 & 2,535 & 3,434 \\
\hline
\end{tabular}

Fuente: Elaboración propia

Figura 1. Perspectiva de las actitudes de los estudiantes

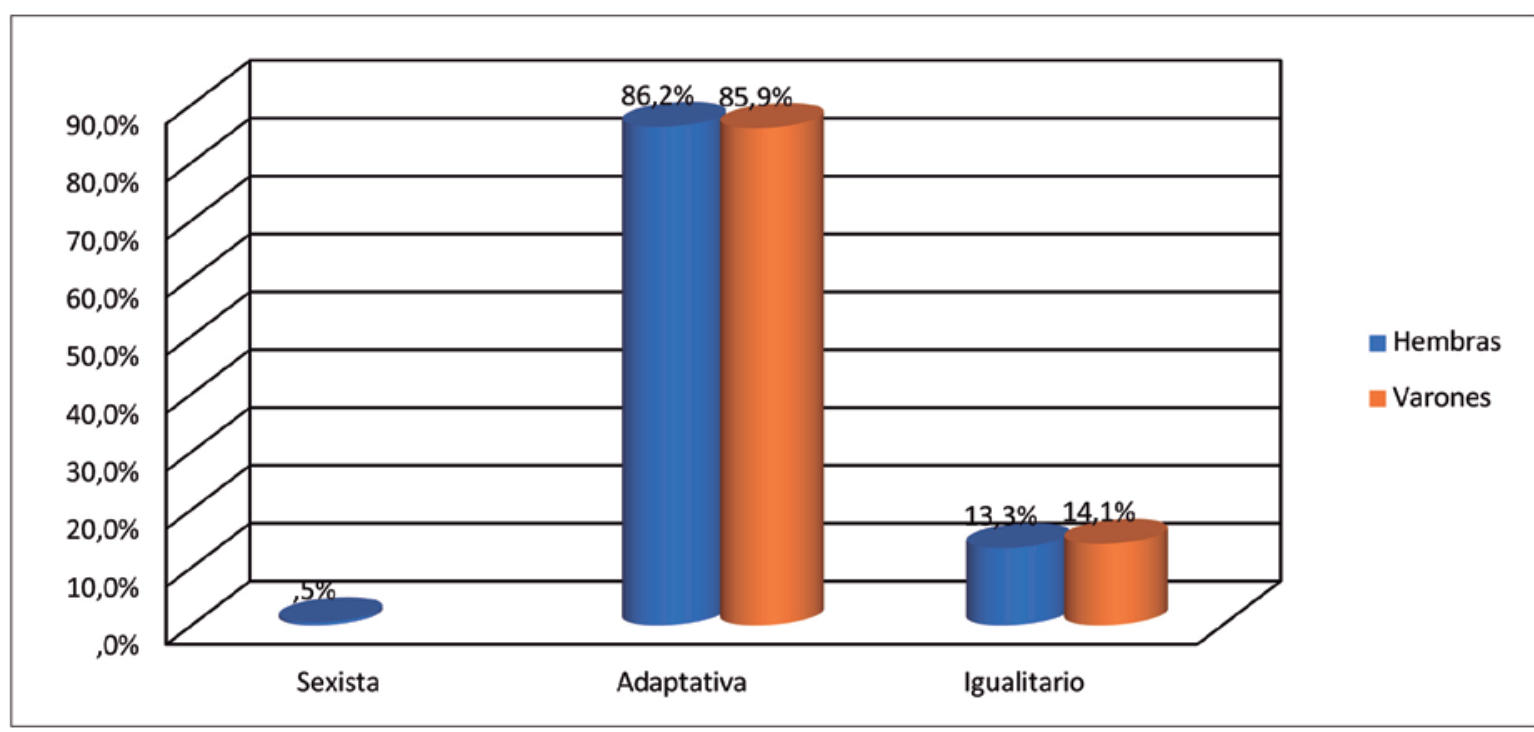

\section{Fuente: Elaboración propia.}

En la tabla 3 se pueden observar los resultados del análisis de correlación no paramétrica de Spearman's. Existe una correlación altamente significativa entre la perspectiva de género y la interacción social, a un nivel de significancia de 0,01 . Esto explica que ambas variables están altamente asociadas; por lo tanto, los resultados en los indicadores de la perspectiva de género muestran una actitud adaptativa que incide directamente en un trato de igualdad y equidad en la interacción social de los estudiantes. 
Tabla 3. Correlación no paramétrica de Spearman's

\begin{tabular}{|c|c|c|c|c|}
\hline & & & $\begin{array}{l}\text { Perspectiva de } \\
\text { género }\end{array}$ & $\begin{array}{c}\text { Interacción } \\
\text { social }\end{array}$ \\
\hline \multirow{6}{*}{ rho de Spearman's } & \multirow{3}{*}{ Perspectiva de género } & Coeficiente de Correlación & 1,000 &, $370^{* *}$ \\
\hline & & Sig. (2-tailed) & . & ,000 \\
\hline & & $\mathrm{N}$ & 259 & 259 \\
\hline & \multirow{3}{*}{ Interacción social } & Coeficiente de Correlación &, $370^{\star *}$ & 1,000 \\
\hline & & Sig. (2-tailed) & ,000 & . \\
\hline & & $\mathrm{N}$ & 259 & 259 \\
\hline
\end{tabular}

** La Correlación es significativa en el nivel 0.01 (2-tailed).

Fuente: Elaboración propia.

\section{Discusión}

La investigación conllevó a realizar un análisis estructural desde la diversidad de características que pueden ser generadas por una muestra representativa de alumnos. Se pudo constatar una diferencia marcada de la perspectiva de género con respecto al sexo. La información permitió construir un análisis de los indicadores de las variables:

Estereotipo: Los estudiantes mantienen una alta perspectiva con una tendencia a moderado, sobre los estereotipos acerca de las generalizaciones y creencias; lo cual es entendible porque en la etapa adolescente se adquiere paulatinamente un conocimiento más preciso de estos aspectos. Al respecto, Andrade-Zurita et al. (2017) afirman que estas prácticas sociales, estos modelos ejercitados, ideas creadas, discursos y representaciones culturales, son aceptados en la moral y ética por las comunidades y simultáneamente promovidos y reproducidos por las instituciones escolares.

Discriminación: Aquí se imponen los estereotipos; surge cuando las diferencias entre géneros se convierten en desigualdad. Sin embargo, en los resultados, los estudiantes aceptan tener amigos homosexuales, lo que se manifiesta con un grado de aceptación hacia la igualdad; pero se disocia en actividades que son consideradas para mujeres (tareas de cuidado), lo que permite infe- rir que existe una alta percepción en las establecidas al rol estereotipado para los varones $y$, moderada para el comportamiento que es adecuado para mujeres. A ese respecto, Andrade-Zurita et al. (2017), refieren que existen apreciaciones de los alumnos acerca de la conducta social representada por mujeres u hombres; y que estos relacionan la discriminación sociocultural a la perspectiva de género. Para Silva- Diverio (2016) los adolescentes, en su comportamiento, se interconectan y apoyan en actividades propias de su sexo. En el caso de las hembras, nuevamente, se evidencian actitudes relacionadas con labores de atención a las personas, la comprensión y los sentimientos; mientras que los varones destacan en la capacidad de obrar con fuerza física, medir la superioridad de uno con el otro y mostrar su aspecto varonil.

Violencia: La información mostró una actitud claramente sexista e influida socialmente por estereotipos que abundan en considerar, el sometimiento social del hombre sobre la mujer. Se acepta la vulnerabilidad de la mujer, creencia compartida por la misma muestra de hembras. De la Osa et al. (2013) reportaron que, los adolescentes, en un importante número, son tolerantes y no están de acuerdo con acciones violentas; sin embargo, persisten momentos en los cuales su comportamiento amerita respuestas agresivas basadas en el papel que cumplen como 
hombres. Por tanto, se debe evitar a como dé lugar la agresión a la mujer y hacer entender a los estudiantes, como lo afirman Jiménez y Estévez (2017) "la igualdad de género propicia el desarrollo en las sociedades (p. 254).

Sexo: Los adolescentes mantienen una perspectiva sexista ante la figura femenina $y$ masculina en la sociedad, persistiendo la postura de vulnerabilidad hacia la mujer por el hecho de serlo. Se observa que las posiciones sexistas y adaptativas están relacionadas al carácter social y estereotipado, que les dan a los indicadores involucrados en esta dimensión; son adquiridos en un proceso en los que se integran, la cultura, el entorno social, el grupo familiar y la escuela. Segato (2016) afirma que, con la edad, las diferencias signadas en el comportamiento entre varones y hembras cada vez es mayor y repercuten negativamente en sus interrelaciones; por lo que se debe educar a la población no solo con medidas explícitas, sino también a través de medidas indirectas que propicien valores y actitudes positivos en los comportamientos de discriminación, para hacerlos visibles y eliminarlos.

Equidad: La equidad se encuentra soslayada; se observa una sobrevaloración sexista ante este indicador. Sin embargo, los estudiantes son más sensibles acerca de que las hembras superan académicamente a los varones $y$, en el campo deportivo y social, consideran que hay igualdad en ambos sexos en la práctica de juegos. Al respecto, Ruiz-Ramírez y Ayala-Carrillo (2016) explican la importancia de concientizar sobre la equidad de género para transformar la forma de pensar, ante los roles que mujeres y hombres, cumplen en el desarrollo social y económico.

Igualdad: Los adolescentes han aceptado que existen otras formas de masculinidad $y$ feminidad y que pueden convivir en ambientes educativos en igualdad de condiciones, pero se observó una tendencia a asumir que no existe igualdad en ciertas actividades estereotipadas tanto de hombres y mujeres. En este sentido, Villegas (2018) demostró que existe una marcada desigualdad entre las estudiantes en relación con los varones que gozan de mayor consideración en el ámbito social. Al respecto, Vega et al. (2019) afirman que para lograr un desarrollo equilibrado y productivo se deben implementar acciones que propicien la igualdad entre varones y hembras, así como la formulación de un cuerpo de políticas que regulen y fomenten la participación equitativa con miras a promover una educación inclusiva y respetuosa del otro. Los adolescentes son más considerados cuando se habla de igualdad, eso ayuda a "reducir las brechas de género en cuanto a oportunidades y derechos" (UNESCO, 2015, p.14).

De acuerdo con los puntos de corte definidos, la muestra manifestó perspectivas adaptativas que inciden directamente en un trato de igualdad y equidad, en la interacción social de los estudiantes. El análisis de correlación evidenció un nivel alto de significancia entre las variables.

\section{Conclusiones}

La perspectiva de género que tienen los adolescentes actuales ha cambiado considerablemente en comparación con la de hace veinte años. Aun cuando en Ecuador continúan prevaleciendo actitudes arcaicas como el machismo y la homofobia, las tendencias han variado a favor de la igualdad de género y condiciones.

En referencia a los factores que inciden en la perspectiva de género del adolescente para el desarrollo social dentro de ambientes escolares en los niveles de Educación General Básica Superior y Bachillerato se infiere que los sociales, religiosos, ideológicos, políticos, históricos, económicos y culturales, prevalecen en los estereotipos, la discriminación, la violencia y el sexo.

Los participantes del estudio con respecto a la actitud adaptativa, mostraron que se han ido adecuando a las situaciones que les deviene el presente; la mayoría lo hace porque no tienen discrepancias en la igualdad de oportunidades. Sin embargo, aún queda un bajo porcentaje que se mantiene firme a las enseñanzas sexistas que le han inculcado desde su núcleo familiar. 
Se evidenció que los alumnos entienden que la mujer es parte fundamental y activa de la sociedad, no solo como madre, sino también como profesional, que ayuda a mejorar el aparato productivo del país; aun así, se mantienen tendencias a ubicarlas en la realización de tareas domésticas.

Los estereotipos sociales se encontraron relacionados con el hecho que los hombres son el sexo fuerte y lideran las actividades grupales, viéndose como muy natural que los padres concedan mayor libertad a los varones. También los resultados evidencian la tendencia a creer que ellos son más capaces para desempeñar tareas técnicas y mecánicas; responsables de mantener económicamente a la familia, y que el presidente del país debe ser un hombre. Las mujeres, son socialmente consideradas mejores estudiantes y más vulnerables físicamente que los hombres.

En general, parte de la actitud de los jóvenes a nuevas circunstancias se ven marcadas por la formación que reciben en los hogares; existe la condición de estigmatizar lo desconocido, pero esas barreras se han ido disminuyendo pues las nuevas tecnologías permiten apreciar y cotejar situaciones similares que se presentan en otros países. De igual forma, la mujer ha ganado terreno en todos los espacios sociales logrando ubicarse en el lugar de la igualdad, donde el sexo o el género no definen la capacidad individual de las personas.

El análisis realizado en esta investigación reafirma la necesidad de proponer acciones centradas en políticas de igualdad, reforzadas con actividades que sensibilicen sobre estos temas, desde el perfil adaptativo del alumno. Resulta necesario cambiar, modificar y suprimir actitudes y conductas sociales que tipifiquen el género relacionados con la esfera privada y tareas asistenciales. Por otra parte, se recomienda evitar la conformación de escenarios donde se permita la violencia de género, a partir de actividades formativas que busquen la convivencia social y la paz.

Es imprescindible que, desde el hogar, los padres y familiares en general aborden temas que se relacionen con el género como condición social, pero desde un sentido reflexivo y de tolerancia; que evite la exclusión o discriminación de los jóvenes que no se identifiquen con su sexo. En las instituciones educativas se debe capacitar y actualizar a los profesores para que puedan aclarar las dudas que los estudiantes formulen y no exista una concepción o una información errada, que genere dudas y confusión. Se deben implementar estrategias dirigidas a desarrollar un proceso educativo desde la coeducación, basado en valores como la tolerancia, la aceptación a nuevas formas de expresar su género, la convivencia armónica, la diversidad, la inclusión, la pluralidad y la autonomía.

\section{Referencias bibliográficas}

Aguilar, Y., Valdez, J., González, N., \& González, S. (2013). Los roles de género de los hombres y las mujeres en el México Contemporáneo. Enseñanza e Investigación en Psicología, 18(2), 207-224. https://bit.ly/2KZwgRP

Andrade-Zurita, S., Rosero-Morales, E., LucasSolórzano, C., Armas-Arias, S., \& NúñezEspinoza, M. (2017). Violencia de género en ambientes escolares: Desigualdades entre los sexos. UTCIENCIA. Ciencia y tecnología al servicio del pueblo, 4(1), 24-36. https://bit.ly/2W1LRqo

Asamblea Constituyente de la República del Ecuador. Constitución de la República del Ecuador. Registro Oficial. No 449. Año II, del 20-102008.

Azorín-Abellán, C.M. (2017). Actitudes hacia la igualdad de género en una muestra de estudiantes de Murcia. Revista Complutense de Educación, 28(1), 45-60. http://dx.doi. org/10.5209/rev_RCED.2017.v28.n1.48715

Beltrán-Gálvez, M. (2012). La otra cara de la moneda: mujeres que practican violencia. Revista Punto Género, (2), 71-92. https://doi.org/10.5354/0719-0417.2012.28365

Benavente, M., \& Valdés, A. (2014). Políticas públicas para la igualdad de género: Un aporte a la autonomía de las mujeres. Comisión 
Económica para América Latina y el Caribe (CEPAL). https://bit.ly/3dlxX87

Bruel, T., Scarparo, H., Calvo, A., Sebastián, J., \& Blanco, A. (2013). Estudio psicosocial sobre las representaciones sociales de género. [A Psychosocial Study about the Social Representations of Gender] Diversitas: Perspectivas en Psicología, 9(2), 243-255. https://doi.org/10.15332/s1794-9998.2013.0002.01

Cabezas, E., Andrade, D., \& Torres, J. (2018). Introducción a la metodología de la investigación científica. ( $1^{\text {a }}$. Ed). Electrónica. Universidad de las Fuerzas Armadas ESPE. https://bit.ly/2xMXHeB

Camarera, M., \& Saavedra, M. (2018). La perspectiva de género en los programas de estudio de las licenciaturas contables administrativas. [The gender perspective in the curriculum of management accountants degrees]. Nóesis: Revista de Ciencias Sociales y Humanidades, 27(54). https://doi.org/10.20983/noesis.2018.2.3

Chávez-Carapia, J. (2017). Percepción de la igualdad de género en jóvenes universitarios. Grupo de investigación Centro de Estudios de la Mujer. 2015-2016. UNAM: Revista de Trabajo Social, 75-90. https://bit.ly/2SLtF2m

Defensoría del Pueblo-Ecuador (2016). Política Institucional de Igualdad de Género (20162019). https://bit.ly/2WWCUyX

Duarte, J., \& García, J. (2016). Igualdad, equidad de género y feminismo, una mirada histórica a la conquista de los derechos de las mujeres. [ Equity, Gender Equality and Feminism, a historical look at the conquest women's rights]. Revista CS, 2(18), 13-38. https://doi.org/10.18046/recs.i18.1960

De la Osa, Z., Andrés, S., \& Pascual, I. (2013). Creencias adolescentes sobre la violencia de género. Sexismo en las relaciones entre adolescentes. European Journal of Investigation in Health, Psychology and Education, 3(3), 265275. https://doi.org/10.30552/ejihpe.v3i3.51

Fondo de las Naciones Unidas para la Infancia (2015). Educación para Todos. Organización de las Naciones Unidas para la Educación, la Ciencia y la Cultura.

Gamboa, M. (2017). Estadística aplicada a la investigación educativa. Dilemas Contemporáneos: Educación, Política y Valores, V(2).
https://bit.ly/2xJBbU1

García-Pérez, R., Rebollo, M.A, Buzón, O., GonzálezPiñal, R., Barragán, R., \& Ruiz, E. (2010). Actitudes del alumnado hacia la igualdad de género. Revista de Investigación, 28(1), 217232. https://bit.ly/2zgDRZN

Guerra-López, R. (2016). Persona, sexo y género. Los significados de la categoría "género" y el sistema "sexo/género" según Karol Wojtyła. Revista de Filosofía Open Insight, 7(12), 139-164. https://bit.ly/2XsvkLL

Guzmán, F. (2015). Violencia de género en adolescentes: Análisis de las perspectivas y de las acciones educativas propuestas por la Junta de Andalucía. (Tesis de doctorado). Universidad de Sevilla. https://bit.ly/3fnU0gm

Hernández, R., Fernández, R., \& Baptista, P. (2014). Metodología de la investigación (6a. ed.). Mc Graw Hill.

Javaloy, F., Espelt, E., \& Rodríguez, Á. (2016). Comportamiento colectivo y movimientos sociales en la era global. En J. Morales, E. Gaviria, M. Moya, \& I. Cuadrado (Coords.), Psicología social, 641-691. Mc Graw Hill. https://bit.ly/2AavSOH

Liscano, D. (2016). Representaciones sociales sobre las personas LGBTI en la universidad: perspectivas del profesorado y alumnado. Revista Nacional e Internacional de Educación Inclusiva, 9(3), 231-249. https://bit.ly/2WduByn

Lozano, A.V. (2014). Teoría de Teorías sobre la Adolescencia. [Theory of Theories about Adolescence]. Última década, 22(40), 11-36. https://dx.doi.org/10.4067/S0718-22362014000100002

Medina, V., Coronilla, U., \& Bustos, E. (2015). La discriminación dentro del salón de clases. [Discrimination in the classromm]. RIDE Revista Iberoamericana para la Investigación $y$ el Desarrollo Educativo, 6(11), 855-867. https://doi.org/10.23913/ride.v6i11.134

Meza, J., \& Páez, R. (2016). Familia, escuela y desarrollo humano: Rutas de investigación educativa. ( $1^{\text {a }}$ ed). Kimpres S.A.S. https://bit.ly/36qnYfw

Organización de las Naciones Unidas (1995). La Declaración de Beijing: IV Conferencia Mundial sobre Mujeres. IV Conferencia 
Mundial sobre las mujeres. Beijing: N.N.U.U. https://bit.ly/3b91FMs

Organización de las Naciones Unidades Mujeres (2014). Declaración Política y documentos de Beijing +5. ONUM. https://bit.ly/2Wegxoz

Organización de las Naciones Unidas para la Educación la Ciencia y la Cultura (2015). Igualdad de género. Patrimonio y creatividad. https://bit.ly/3cYW8tu

Ortiz-Jiménez, J.G., Ramírez-Garzón, M.I., Moreno-F., M., \& Martínez-Morales, E. M. (2018). Las rutas de las emociones: Sujetos e instituciones en tránsito a la paz. Taller de Edición Rocca. https://bit.ly/2WAEqpg

Rodríguez, P., Matud, M., \& Álvarez, J. (2017). Género y calidad de vida en la adolescencia. Journal of Behavior, Health \& Social Issues, 9(2), 89-98. https://doi.org/10.1016/j.jbhsi.2017.11.001

Ruiz-Ramírez, R., \& Ayala-Carrillo, M. (2016). Violencia de género en instituciones de educación. Ra Ximhai, 21-32. https://doi.org/10.35197/rx.12.01.2016.01.rr

Secretaría Nacional de Planificación y Desarrollo. Consejo Nacional de Planificación (2017). Plan Nacional Toda una Vida 2017-2021. Quito. Ecuador. Resolución No. CNP-0032017 del 22-09-2017. https://bit.ly/3cYDBgV

Segato, R. (2016). La guerra contra las mujeres. Traficantes de sueños: Mapas. https://bit.ly/2LVoHMk

Serret, E. (2016). Hacia una redefinición de las identidades de género. GénEro ơs, Revista de investigación y divulgación sobre los estudios de género, 18(9), 71-97. https://bit.ly/3fr6Hai

Silva-Diverio, I. (2016). La adolescencia y su interrelación con el entorno. Instituto de la Juventud (INJUVE). https://bit.ly/2WXA6lm
Solís-Sabanero, A. (2016). La perspectiva de género en la educación. En J.A. Trujillo-Holguín y J.L. García-Leos (Coords.), Desarrollo profesional docente: reforma educativa, contenidos curriculares y procesos de evaluación, 97-107. https://bit.ly/2SLNXsx

Suárez, P., \& Vélez, M. (2016). El papel de la familia en el desarrollo social del niño: una mirada desde la afectividad, la comunicación familiar y estilos de educación parental. [The role of the family in social development of child: a look from affectivity, family communication and education] Psicoespacios, 12(20),173197. https://doi.org/10.25057/issn.2145-2776

Torres, L. (2018). Interiorización de los estereotipos de género en la sociedad argentina y el ideal de belleza en los mensajes publicitarios [Tesis de doctorado, Universidad Complutense de Madrid]. https://bit.ly/2SPgCgi

Trejo-Sirvent, M.L., Llaven-Coutiño, G., \& Pérez y Pérez, H.C. (2015). El enfoque de género en la Educación. Atenas, 4(32), 49-61. https://bit.ly/2Wb43xI

Vega, A., García, S., Candela, B., Santos, J., Díaz, M., \& Torrado, E. (2019). De la teoría a la práctica educativa en igualdad. [From theory to educational practice in equality]. Revista de la ULL, 31-50.

https://doi.org/10.25145/b.innovaull.2019.002

Villegas, V. (2018). Concepciones de género y convivencia escolar en los y las estudiantes de la Unidad Educativa CMDT. César Endara Peña Herrera. [Conceptions of gender and school coexistence in the students of the Educational Institution CMDT César Endara Peña Herrera]. Retos de la Ciencia, 2(2), 139154. https://bit.ly/2WGcUGZ 